Check for updates

Cite this: RSC Adv., 2017, 7, 40705

\title{
High-flux polyamide reverse osmosis membranes by surface grafting 4-(2-hydroxyethyl)morpholine
}

\author{
Ruizhi Pang (D) and Kaisong Zhang*
}

Surface modification is a common approach to improve the desalination performance of polyamide (PA) reverse osmosis $(\mathrm{RO})$ membranes. Nevertheless, the water flux normally decreases due to the additional hydraulic resistance of the surface modification layer. Surface grafted PA RO membrane with 4-(2hydroxyethyl)morpholine was first fabricated to improve water flux in this study. 4-(2-Hydroxyethyl) morpholine was in situ grafted onto the nascent PA membrane by the reaction between the $-\mathrm{OH}$ group of 4-(2-hydroxyethyl)morpholine and the unreacted - $\mathrm{COCl}$ groups of the nascent PA membrane and residual trimesoyl chloride. The surface grafted RO membranes were characterized by $\mathrm{X}$-ray photoelectron spectrometry, field emission scanning electron microscopy, atomic force microscopy and contact angle measurement. The surface grafting conditions, including 4-(2-hydroxyethyl)morpholine dipping time, heat treatment time, and 4-(2-hydroxyethyl)morpholine concentration, were optimized by characterizing the desalination performance under brackish water desalination conditions. The resulting surface grafted membrane exhibited a water flux of $125.71 \mathrm{~L} \mathrm{~m}^{-2} \mathrm{~h}^{-1}$ and a salt rejection of $98.6 \%$. The surface grafted membrane surpassed the control polyamide membrane with $41.9 \%$ increase in the water flux. Our results demonstrated that surface modification by in situ grafting 4-(2-hydroxyethyl)morpholine is a promising approach to fabricate high-flux RO membranes.

Received 10th June 2017

Accepted 14th August 2017

DOI: $10.1039 / c 7 r a 06486 a$

rsc.li/rsc-advances by Perreault et al. ${ }^{29}$ Graphene oxide functionalization improved the antimicrobial performance significantly. Yang et al. grafted and deposited pyridine-based zwitterionic copolymers onto TFC RO membranes via initiated chemical vapor deposition..$^{30}$ The antifouling performance and chlorine stability were improved by ultrathin zwitterionic coatings. The aforementioned studies demonstrated that surface modification was an effective method to improve the salt rejection and antifouling performance of PA TFC RO membrane. However, the water flux normally decreased due to the additional hydraulic resistance of surface modification layer. ${ }^{3}$

4-(2-Hydroxyethyl)morpholine contains a hydrophilic morpholine portion and the reactive $-\mathrm{OH}$ group which can react with TMC during the IP process. Zhao et al. fabricated the highflux RO membrane by incorporating 4-(2-hydroxyethyl)morpholine as hydrophilic additive into the amine solution during the IP process. ${ }^{5}$ The water flux was enhanced significantly. However, the majority of 4-(2-hydroxyethyl)morpholine was embedded in the PA bulk. ${ }^{3}$

Herein, for the first time, the surface grafted PA RO membrane with 4-(2-hydroxyethyl)morpholine was fabricated to improve water flux. 4-(2-Hydroxyethyl)morpholine was in situ grafted onto the nascent PA membrane by the reaction between the -OH group of 4-(2-hydroxyethyl)morpholine and the unreacted -COCl groups of nascent PA membrane and residual trimesoyl chloride during the RO membrane preparation process (Fig. 1). The surface grafted RO membranes were 
<smiles>CCC(=O)c1cc(C(=O)Cl)cc(C(=O)Nc2cccc(NC(=O)c3cc(C(=O)Nc4cccc(NC)c4)cc(C(=O)Nc4cccc(NC(=O)c5cc(C(=O)Cl)cc(C(=O)Cl)c5)c4)c3)c2)c1</smiles>

Nascent Polyamide 4-(2-Hydroxyethyl) Morpholine<smiles>CNc1cccc(NC(=O)c2cc(C(=O)Nc3cccc(NC(=O)c4cc(C(=O)Nc5cccc(N)c5)cc(C(=O)OCCN5CCOCC5)c4)c3)cc(C(=O)OCCN3CCOCC3)c2)c1</smiles>

Surface Grafted Polyamide RO Membrane

Fig. 1 Grafting reactions of 4-(2-hydroxyethyl)morpholine onto the nascent polyamide membrane.

characterized by X-ray photoelectron spectrometer, field emission scanning electron microscopy, atomic force microscopy and contact angle goniometer. Finally, the surface grafting conditions, including 4-(2-hydroxyethyl)morpholine dipping time, heat treatment time, and 4-(2-hydroxyethyl)morpholine concentration, were optimized by characterizing the desalination performance of surface modified PA RO membranes.

\section{Experimental section}

\subsection{Materials}

$m$-Phenylenediamine (MPD), ( \pm )-camphor-10-sulfonic acid (CSA), trimethylamine (TEA) and 4-(2-hydroxyethyl)morpholine were purchased from Aladdin (Shanghai, China). Trimesoyl chloride (TMC) was received from TCI (Shanghai, China). Isopar $\mathrm{G}$ was obtained from ExxonMobil Chemical (Shanghai, China). Isopropanol (IPA) and sodium chloride ( $\mathrm{NaCl}$ ) were purchased from Sinopharm (Shanghai, China).

\subsection{Preparation of surface grafted PA TFC RO membrane}

The schematic diagram for fabrication process of surface grafted membrane was shown in Fig. 2. The polysulfone support was first immersed in an amine aqueous solution containing 2 $\mathrm{wt} \%$ MPD and $5 \mathrm{wt} \%$ CSA-TEA salt for $6 \mathrm{~min}$. After removing the excess amine droplets by tissue papers, a $0.1 \mathrm{wt} \% \mathrm{TMC} / \mathrm{Isopar} \mathrm{G}$ solution was poured onto the amine saturated support to react for $20 \mathrm{~s}$. The excess TMC solution was then drained by standing vertically for $1 \mathrm{~min}$. Next, the nascent PA membrane was immediately soaked in a $0.5-2 \mathrm{wt} \%$ 4-(2-hydroxyethyl)morpholine in IPA solution of $\mathrm{pH} 9.5$ adjusted with TEA for 10-50 s. Finally, the membrane was cured in the oven at $90{ }^{\circ} \mathrm{C}$ for $4-$ 8 min.

With the exception of the 4-(2-hydroxyethyl)morpholine dipping step, the preparation process of control PA membrane without IPA solution treatment is identical to that of surface grafted membrane. In addition, the PA membrane only treated

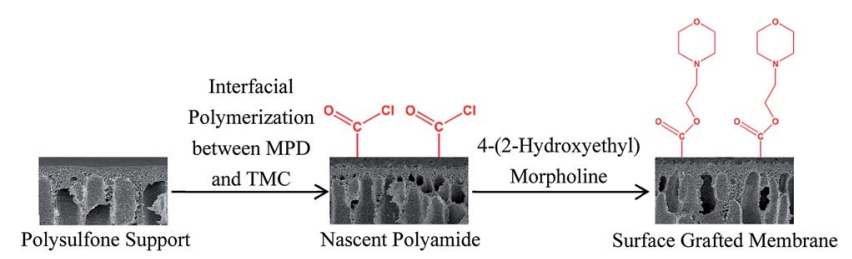

Fig. 2 Schematic diagram for the fabrication process of surface grafted membrane. 
by IPA solution without 4-(2-hydroxyethyl)morpholine was also prepared under the same preparation conditions.

\subsection{Characterization of surface grafted PA TFC RO membrane}

The successful grafting of 4-(2-hydroxyethyl)morpholine on membrane surface was examined by X-ray photoelectron spectrometer (XPS, ESCALAB 250). The membrane morphologies were assessed by field emission scanning electron microscopy (FESEM, HITACHI S-4800) and atomic force microscopy (AFM, Agilent 5500). The contact angles of both control polyamide membrane and surface grafted membrane were measured by contact angle goniometer (Krüss DSA 30).

The feed solution of $2000 \mathrm{ppm} \mathrm{NaCl}$ was used to evaluate the water flux and salt rejection in a cross-flow stainless steel cell (Sterlitech) under $1.55 \mathrm{MPa}$ at room temperature. The membrane with an active area of $42 \mathrm{~cm}^{2}$ were compacted for $3 \mathrm{~h}$ to reach the steady state. After that, the water flux and salt rejection were determined, according to the reported method in the literature.$^{4-6}$ All the water flux and salt rejection results are the average values of at least three membrane samples.

\section{Results and discussion}

\subsection{Characterization of surface grafted membrane}

The elemental composition of membrane surface was analyzed by XPS. The oxygen-to-carbon $(\mathrm{O} / \mathrm{C})$ ratio of 4-(2-hydroxyethyl) morpholine $(1 / 3)$ is higher than that of control polyamide membrane $(0.165)$, thus the oxygen-to-carbon $(\mathrm{O} / \mathrm{C})$ ratio of surface grafted membrane is expected to be increased. As presented in Table 1, the $\mathrm{O} / \mathrm{C}$ ratio of surface grafted membranes increased with the increase in 4-(2-hydroxyethyl)morpholine concentration. The grafting degree of surface grafted membranes with $0.5 \%, 1.0 \%, 1.5 \%$ and $2.0 \%$ 4-(2-hydroxyethyl) morpholine concentration was $15.8 \%, 22.4 \%, 32.1 \%$ and $37.6 \%$, respectively. The XPS analysis verified successful grafting of 4-(2-hydroxyethyl)morpholine on membrane surface.

The effects of surface grafting on membrane morphologies were investigated by SEM and AFM. The SEM images of both membrane surface and cross section morphology are shown in Fig. 3. The ridge-and-valley structure was observed in both control polyamide membrane and surface grafted membrane. However, the thickness of surface grafted membrane decreased. In the previously reported literature, ${ }^{31,32} n$-hexane solution was used to wash away the residual reagents of nascent PA membrane surface during the fabrication process. Likewise, some residual reagents on nascent PA membrane surface were washed away by the IPA modification solution. Therefore, the thickness of the active layer of the modified membrane was thinner than that of the control PA membrane.

The surface roughness of both control polyamide membrane and surface grafted membrane with $0.5 \mathrm{wt} \%$ 4-(2-hydroxyethyl) morpholine was measured by AFM. As shown in Fig. 4, the mean roughness $\left(R_{\mathrm{a}}\right)$ decreased from $45.4 \mathrm{~nm}$ for control polyamide membrane to $24.9 \mathrm{~nm}$ for surface grafted membrane with 0.5 wt\% 4-(2-hydroxyethyl)morpholine. The AFM results demonstrated the membrane surface was smoothened by surface grafting 4-(2-hydroxyethyl)morpholine.

The membrane surface hydrophilicity was assessed by water contact angle. As presented in Fig. 5, the water contact angle of control PA membrane was $90.7^{\circ}$, which is consistent with the contact angle value of hand-cast PA membranes reported in the literature. ${ }^{33,34}$ After surface grafting 4-(2-hydroxyethyl)morpholine, the water contact angle reduced to $81.8^{\circ}$. The relatively hydrophilic 4-(2-hydroxyethyl)morpholine grafted onto the PA surface was responsible for the increased hydrophilicity.

\subsection{Effects of 4-(2-hydroxyethyl)morpholine concentration on desalination performance}

The effects of 4-(2-hydroxyethyl)morpholine concentration on desalination performance were depicted in Fig. 6 . The flux was improved considerably from $88.57 \mathrm{~L} \mathrm{~m}^{-2} \mathrm{~h}^{-1}$ for control PA membrane to $125.71 \mathrm{~L} \mathrm{~m}^{-2} \mathrm{~h}^{-1}$ for surface grafted membrane with 0.5 wt $\%$ 4-(2-hydroxyethyl)morpholine $(15.8 \%$ grafting degree), while the salt rejection changed slightly from $98.8 \%$ to 98.6\%. As presented in Table 1, the carbon-to-nitrogen $(\mathrm{C} / \mathrm{N})$ ratio increased by surface grafting 4-(2-hydroxyethyl)morpholine. The increase of $\mathrm{C} / \mathrm{N}$ ratios suggests less cross-linking for surface grafted membrane, ${ }^{35,36}$ thus more free volume was created. Moreover, the membrane hydrophilicity was improved and the membrane thickness was reduced by surface grafting. Therefore, the water flux increased significantly. However, with further increase in 4-(2-hydroxyethyl)morpholine concentration, the flux decreased due to the additional hydraulic resistance of surface grafting layer. As a result, $0.5 \%$ was determined as the optimal 4-(2-hydroxyethyl)morpholine concentration.

Table 1 Relative surface atomic concentration of the control polyamide membrane and surface grafted membrane with different 4-(2hydroxyethyl)morpholine concentration

\begin{tabular}{|c|c|c|c|c|c|c|}
\hline \multirow[b]{2}{*}{ Sample } & \multicolumn{3}{|c|}{ Atoms percent (mol\%) } & \multirow[b]{2}{*}{$\mathrm{O} / \mathrm{C}$} & \multirow[b]{2}{*}{$\mathrm{C} / \mathrm{N}$} & \multirow[b]{2}{*}{ Grafting degree (\%) } \\
\hline & $\mathrm{C}$ & $\mathrm{O}$ & $\mathrm{N}$ & & & \\
\hline Surface grafted membrane- 0.5 & 73.84 & 14.08 & 12.09 & 0.191 & 6.11 & 15.8 \\
\hline Surface grafted membrane- 1.0 & 73.39 & 14.83 & 11.78 & 0.202 & 6.23 & 22.4 \\
\hline Surface grafted membrane-1.5 & 72.68 & 15.82 & 11.50 & 0.218 & 6.32 & 32.1 \\
\hline
\end{tabular}




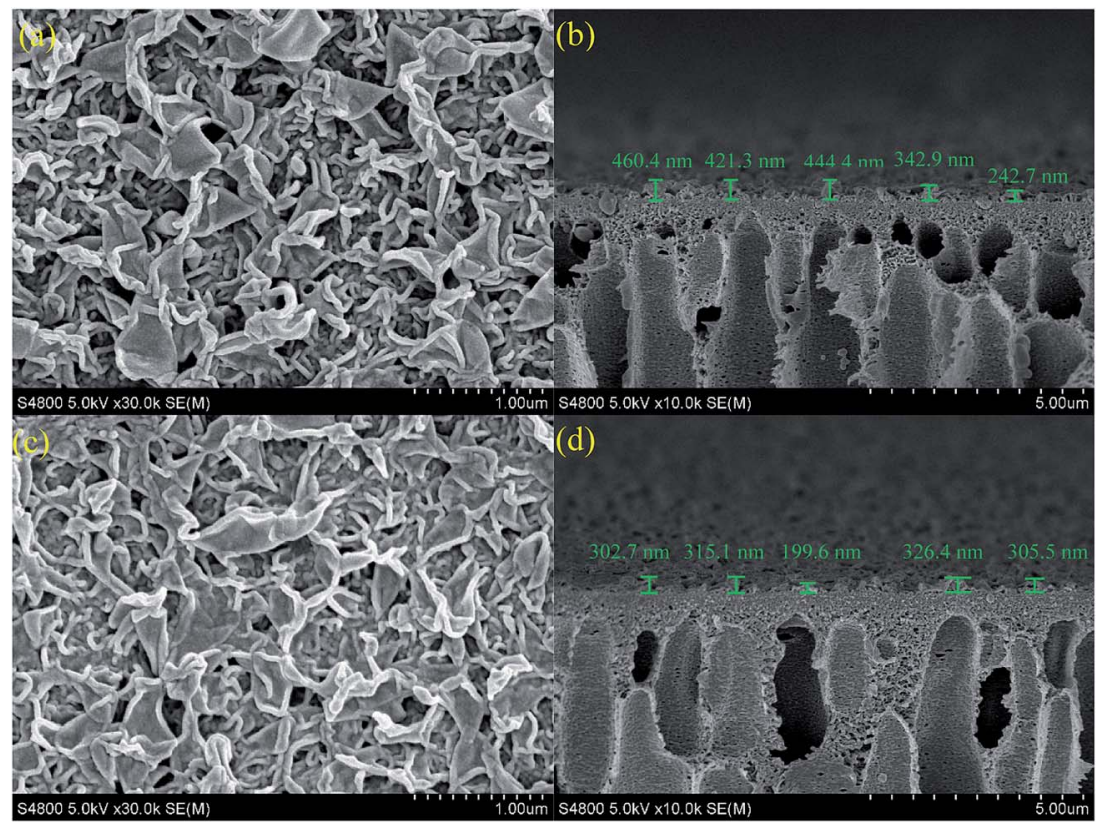

Fig. 3 SEM images for (a) the surface of control polyamide membrane, (b) the cross section of control polyamide membrane, (c) the surface of surface grafted membrane with $0.5 \mathrm{wt} \%$ 4-(2-hydroxyethyl)morpholine and (d) the cross section of surface grafted membrane with $0.5 \mathrm{wt} \% 4$ (2-hydroxyethyl)morpholine.

(a)

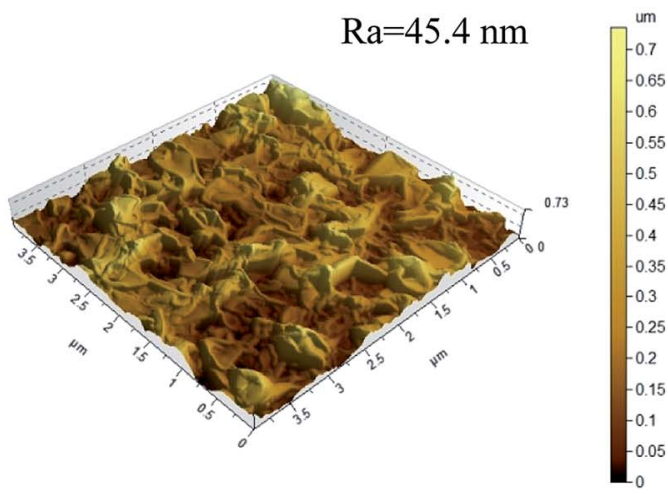

(b)

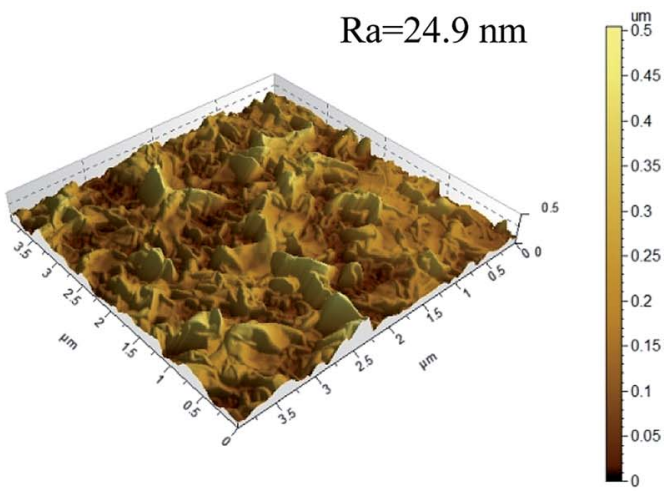

Fig. 4 AFM images of (a) control polyamide membrane and (b) surface grafted membrane with 0.5 wt\% 4-(2-hydroxyethyl)morpholine.

\subsection{Effects of 4-(2-hydroxyethyl)morpholine dipping time on desalination performance}

The effects of 4-(2-hydroxyethyl)morpholine dipping time on desalination performance were investigated. As shown in Fig. 7, the flux increased from $117.14 \mathrm{~L} \mathrm{~m}^{-2} \mathrm{~h}^{-1}$ for $10 \mathrm{~s}$ dipping time

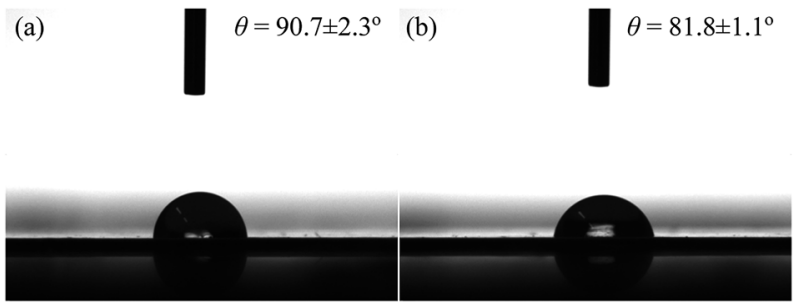

Fig. 5 Water contact angle of (a) control polyamide membrane and (b) surface grafted membrane with $0.5 \mathrm{wt} \%$ 4-(2-hydroxyethyl)morpholine. to $125.71 \mathrm{~L} \mathrm{~m}^{-2} \mathrm{~h}^{-1}$ for $20 \mathrm{~s}$ dipping time, while the salt rejection changed slightly. A longer 4-(2-hydroxyethyl)morpholine dipping time allows more -OH group of 4-(2-hydroxyethyl) morpholine to react with the $-\mathrm{COCl}$ group of nascent PA membrane, which creates more free volume and increases the membrane hydrophilicity. Therefore, the water flux increased. However, the flux decreased by further increasing dipping time. The flux decrease was attributed to the additional hydraulic resistance of surface grafting layer. As a result, $20 \mathrm{~s}$ was selected as the optimal 4-(2-hydroxyethyl)morpholine dipping time.

\subsection{Effects of heat treatment time on desalination performance}

Heat treatment was used to promote the additional crosslinking reactions and expedite the complete removal of Isopar $\mathrm{G}$ and IPA. ${ }^{37}$ The effects of heat treatment time on desalination 


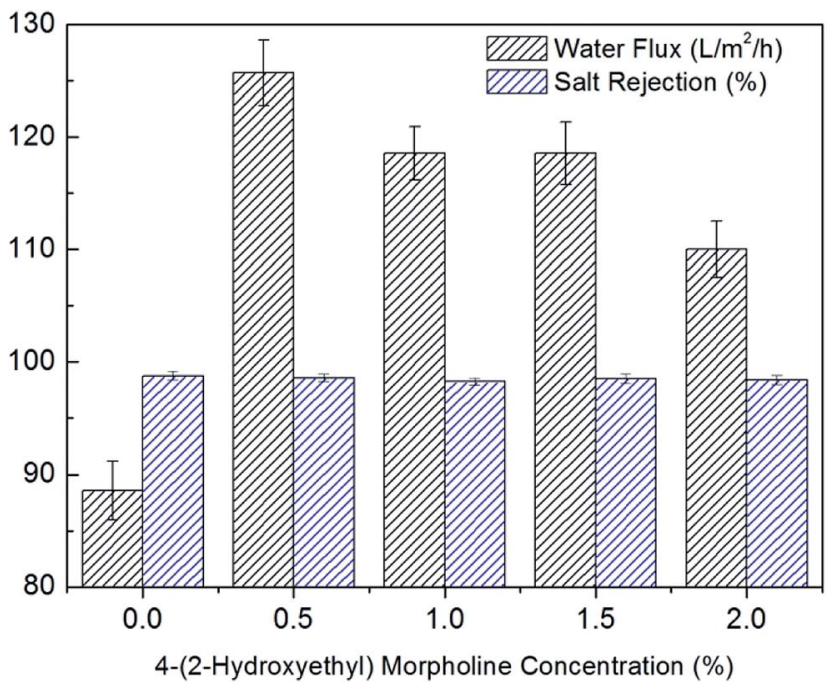

Fig. 6 Effects of 4-(2-hydroxyethyl)morpholine concentration on desalination performance (20 s dipping time and $6 \mathrm{~min}$ heat treatment time).

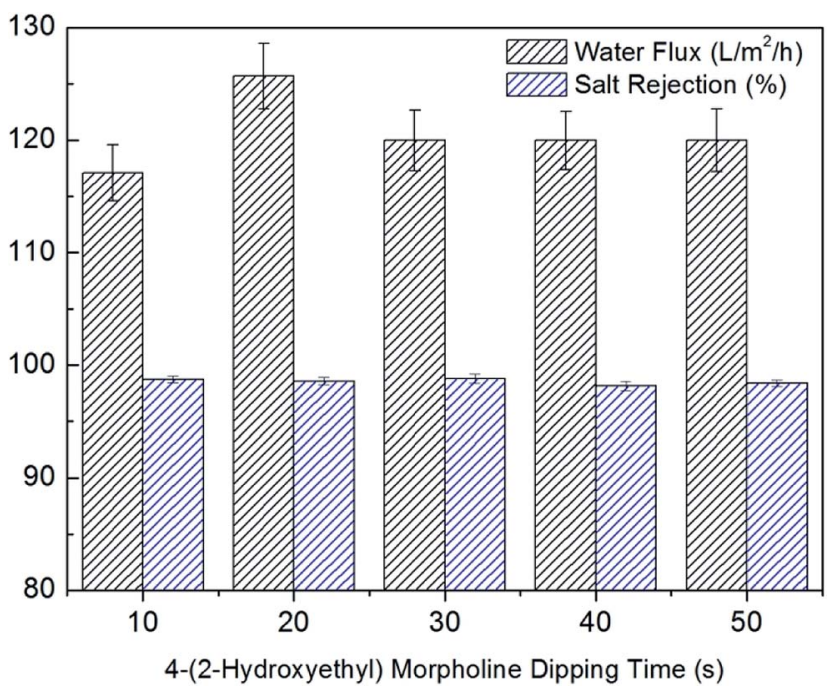

Fig. 7 Effects of 4-(2-hydroxyethyl)morpholine dipping time on desalination performance $(6$ min heat treatment time and $0.5 \mathrm{wt} \%$ 4(2-hydroxyethyl)morpholine).

performance were shown in Fig. 8. The optimal flux was received as the heat treatment was $6 \mathrm{~min}$. The surface grafted membrane showed a water flux of $125.71 \mathrm{~L} \mathrm{~m}^{-2} \mathrm{~h}^{-1}$ and a salt rejection of $98.6 \%$. When the heat treatment was less than $6 \mathrm{~min}$, the cross-linking reactions were not complete. After $6 \mathrm{~min}$, the polysulfone support was destroyed and the water flux decreased..$^{37,38}$ As a result, 6 min was selected as the optimal heat treatment time.

\subsection{Effect of IPA on desalination performance}

The PA membrane only treated by IPA solution without 4-(2hydroxyethyl)morpholine showed a water flux of $102.86 \mathrm{~L} \mathrm{~m}^{-2}$ $\mathrm{h}^{-1}$ and a salt rejection of $97.9 \%$. The PA membrane only

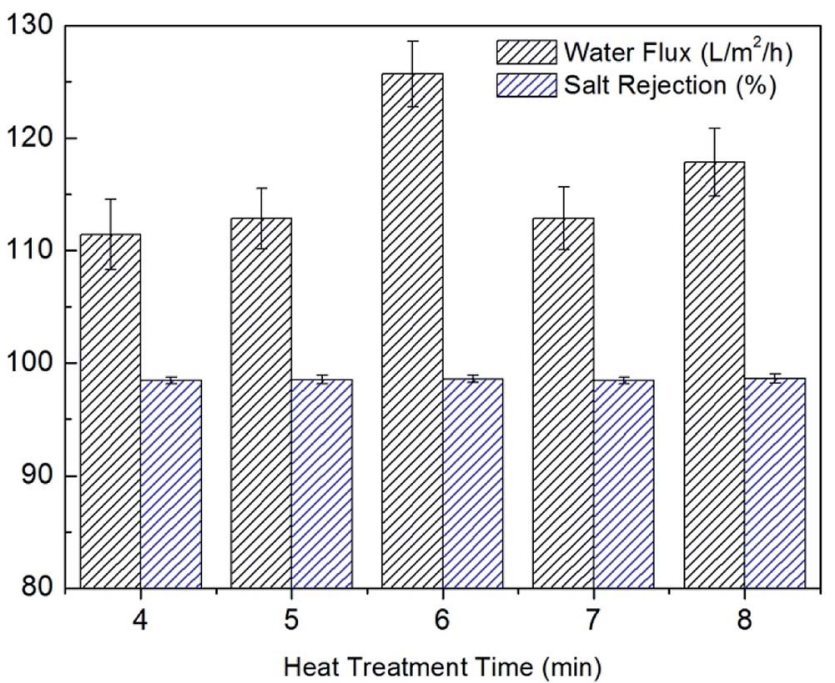

Fig. 8 Effects of heat treatment time on desalination performance (20 s dipping time and $0.5 \mathrm{wt} \%$ 4-(2-hydroxyethyl)morpholine).

treated by IPA solution surpassed the control polyamide membrane with $16.1 \%$ increase in the water flux. However, the salt rejection decreased dramatically from $98.8 \%$ to $97.9 \%$. Some residual reagents on nascent PA membrane surface were washed away by IPA solution, thus the water flux increased and salt rejection decreased. In addition, surface grafted membrane with 0.5 wt\% 4-(2-hydroxyethyl)morpholine surpassed the PA membrane only treated by IPA solution with $22.2 \%$ increase in the water flux, while the salt rejection increased significantly from $97.9 \%$ to $98.6 \%$. Compared to IPA solution without 4-(2hydroxyethyl)morpholine, the IPA solution with 4-(2-hydroxyethyl)morpholine provided not only hydrophilicity for improving water flux but also charge repulsion for enhancing salt rejection.

\section{Conclusions}

The novel surface grafted PA RO membrane with significantly improved water flux was successfully fabricated. The successful grafting of 4-(2-hydroxyethyl)morpholine on membrane surface was confirmed by XPS. The membrane surface roughness decreased due to surface grafting 4-(2-hydroxyethyl)morpholine, while the surface hydrophilicity increased. The surface grafting conditions, including 4-(2-hydroxyethyl)morpholine dipping time, heat treatment time, and 4-(2-hydroxyethyl)morpholine concentration, were optimized. The resulting surface grafted membrane showed a water flux of $125.71 \mathrm{~L} \mathrm{~m}^{-2} \mathrm{~h}^{-1}$ and a salt rejection of $98.6 \%$. The surface grafted membrane surpassed the control polyamide membrane with $41.9 \%$ increase in the water flux.

\section{Conflicts of interest}

There are no conflicts to declare. 


\section{Acknowledgements}

The authors thank the reviewers for their valuable comments. We acknowledge the National Natural Science Foundation of China (Grant No. 51408581), Natural Science Foundation of Fujian Province (Grant No. 2016J05143), Bureau of International Cooperation, CAS (132C35KYSB20160018) and Bureau of Frontier Sciences \& Education, CAS (QYZDB-SSW-DQC044) for the financial support of this work.

\section{References}

1 M. Elimelech and W. A. Phillip, Science, 2011, 333, 712-717.

2 B. Khorshidi, T. Thundat, B. A. Fleck and M. Sadrzadeh, $R S C$ Adv., 2015, 5, 54985-54997.

3 D. Li, Y. Yan and H. Wang, Prog. Polym. Sci., 2016, 61, 104155.

4 L. Zhao, P. C.-Y. Chang, C. Yen and W. S. W. Ho, J. Membr. Sci., 2013, 425-426, 1-10.

5 L. Zhao, P. C.-Y. Chang and W. S. W. Ho, Desalination, 2013, 308, 225-232.

6 L. Zhao and W. S. W. Ho, J. Membr. Sci., 2014, 455, 44-54.

7 S. S. Shenvi, A. M. Isloor and A. F. Ismail, Desalination, 2015, 368, 10-26.

8 W. J. Lau, S. Gray, T. Matsuura, D. Emadzadeh, J. P. Chen and A. F. Ismail, Water Res., 2015, 80, 306-324.

9 D. Li and H. Wang, J. Mater. Chem., 2010, 20, 4551-4566.

10 H. J. Kim, M. Y. Lim, K. H. Jung, D. G. Kim and J. C. Lee, J. Mater. Chem. A, 2015, 3, 6798-6809.

11 B.-H. Jeong, E. M. V. Hoek, Y. Yan, A. Subramani, X. Huang, G. Hurwitz, A. K. Ghosh and A. Jawor, J. Membr. Sci., 2007, 294, 1-7.

12 H. Huang, X. Qu, X. Ji, X. Gao, L. Zhang, H. Chen and L. Hou, J. Mater. Chem. A, 2013, 1, 11343-11349.

13 T. Wang, L. Dai, Q. Zhang, A. Li and S. Zhang, J. Membr. Sci., 2013, 440, 48-57.

14 L.-F. Liu, Z.-B. Cai, J.-N. Shen, L.-X. Wu, E. M. V. Hoek and C.-J. Gao, J. Membr. Sci., 2014, 469, 397-409.

15 K. P. Lee, T. C. Arnot and D. Mattia, J. Membr. Sci., 2011, 370, $1-22$.

16 V. Kochkodan and N. Hilal, Desalination, 2015, 356, 187-207. 17 J. Mansouri, S. Harrisson and V. Chen, J. Mater. Chem., 2010, 20, 4567-4586.

18 D. Rana and T. Matsuura, Chem. Rev., 2010, 110, 2448-2471.
19 J. Wu, Z. Wang, W. Yan, Y. Wang, J. Wang and S. Wang, J. Membr. Sci., 2015, 496, 58-69.

20 J. Wang, Z. Wang, J. Wang and S. Wang, J. Membr. Sci., 2015, 493, 188-199.

21 G. Ye, J. Lee, F. Perreault and M. Elimelech, ACS Appl. Mater. Interfaces, 2015, 7, 23069-23079.

22 W. Ma, A. Soroush, T. V. A. Luong, G. Brennan, M. S. Rahaman, B. Asadishad and N. Tufenkji, Water Res., 2016, 99, 188-199.

23 H. Li, L. Peng, Y. Luo and P. Yu, $R S C A d v .$, 2015, 5, 9856698575.

24 J. S. Louie, I. Pinnau and M. Reinhard, J. Membr. Sci., 2011, 367, 249-255.

25 Q. Zhang, C. Zhang, J. Xu, Y. Nie, S. Li and S. Zhang, Desalination, 2016, 379, 42-52.

26 A. C. Sagle, E. M. V. Wagner, H. Ju, B. D. McCloskey, B. D. Freeman and M. M. Sharma, J. Membr. Sci., 2009, 340, 92-108.

27 R. Bernstein, S. Belfer and V. Freger, Environ. Sci. Technol., 2011, 45, 3613-3620.

28 R. Bernstein, S. Belfer and V. Freger, Environ. Sci. Technol., 2011, 45, 5973-5980.

29 F. Perreault, M. E. Tousley and M. Elimelech, Environ. Sci. Technol. Lett., 2014, 1, 71-76.

30 R. Yang, H. Jang, R. Stocker and K. K. Gleason, Adv. Mater., 2014, 26, 1711-1718.

31 J. Hu, Z. Lv, Y. Xu, X. Zhang and L. Wang, J. Membr. Sci., 2016, 505, 119-129.

32 J. Hu, Y. Pu, M. Ueda, X. Zhang and L. Wang, J. Membr. Sci., 2016, 520, 1-7.

33 A. Tiraferri, Y. Kang, E. P. Giannelis and M. Elimelech, ACS Appl. Mater. Interfaces, 2012, 4, 5044-5053.

34 X. Lu, S. R.-V. Castrillón, D. L. Shaffer, J. Ma and M. Elimelech, Environ. Sci. Technol., 2013, 47, 12219-12228.

35 M. L. Lind, A. K. Ghosh, A. Jawor, X. Huang, W. Hou, Y. Yang and E. M. V. Hoek, Langmuir, 2009, 25(17), 10139-10145.

36 G. N. B. Baroña, J. Lim, M. Choi and B. Jung, Desalination, 2013, 325, 138-147.

37 W. Xie, G. M. Geise, B. D. Freeman, H.-S. Lee, G. Byun and J. E. McGrath, J. Membr. Sci., 2012, 403-404, 152-161.

38 A. K. Ghosh, B. H. Jeong, X. Huang and E. M. V. Hoek, J. Membr. Sci., 2008, 311, 34-45. 